\title{
Estudo de Caso de Alertas e Recomendações para Educação a Distância Aplicado em Turmas Heterogêneas
}

\section{Case Study of Alerts and Recommendations for Distance Learning Applied to Heterogeneous Classes}

\begin{abstract}
Resumo
Este artigo tem por objetivo apresentar um estudo de caso utilizando alertas e recomendações possivelmente adaptativos para alunos e professores aplicados ao ensino mediado por computador. O estudo contempla apoiar o aluno de maneira simples e objetivo no monitoramento das suas ações tomadas no ambiente virtual de aprendizagem (AVA). A base teórica desta pesquisa está centrada no conceito de turmas heterogêneas, levando em consideração o perfil dos alunos, formação acadêmica, diferentes níveis de conhecimento, estratégias de ensino e sistemas de recomendações. Para tanto, o material didático é preparado prevendo atividades que são disponibilizados através de AVA, tais como MOODLE e TELEDUC. Enfim, utilizou-se técnicas de recomendações para identificar os alunos com baixas interações, medir os níveis de participação e conhecimento dos mesmos, viabilizando a adaptação a construção de grupos potencializados a cooperação, e como resultado se propôs um modelo de recomendação, cuja arquitetura agrega as áreas de pesquisa de tecnologia da informação e da educação.

Palavras-chave: Ensino a distância. Ambiente Virtual de Aprendizagem. Sistema de Recomendação.
\end{abstract}

\section{Abstract}

This work aims to present a case study using alerts and recommendations, certainly adaptable for students and teachers. This study is applied to Intermediate Computer Class. The model aims to support the student in a simple and practical way whiles the monitoring of his action in the virtual environment (AVA). The theoretical background of this research is focused on the concept of heterogeneous classes, taking in to account the student's profile, his academic history, different levels of knowledge, teaching strategies and systems recommendations. Considering that, the instructive material is prepared predicting activities trough the AVA, such as MOODLE and TELEDUC. Finally, in this paper we give some recommendations and alerts with the used techniques recommendations to identify the students with low level of participation and measure the levels of interaction, enabling the adaptation to the creation of potentiating groups the cooperation, and as a result a model is proposed recommendation, whose architecture aggregate the areas of research of information technology and education.

Keywords: Distance education. Virtual environment. Recommender system.

MORAIS, Carlos Tadeu Queiroz; FRANCO, Sérgio Roberto K. Estudo de Caso e Alertas e Recomendação para Educação a Distância Aplicado em Turmas Heterogêneas. Informática na Educação: teoria \& prática, Porto Alegre, v. 14, n. 2, p. 129149, jul./dez. 2011.

\section{Carlos Tadeu Queiroz de Morais \\ Sérgio Roberto K. Franco Universidade Federal do Rio Grande do Sul}

\section{Introdução}

H oje ser professor, não é uma tarefa fácil especialmente quando sua função é procurar entender o grau de dificuldade de cada estudante dentro do espaço virtual, tendo como desafio abordar novas metodologias de ensino para turmas com diferentes níveis de conhecimento e aprendizagem.

É sabido que não é tão simples, pois requer aplicações de diversas técnicas e métodos de ensino, mas mesmo assim não é possível alcançar a todos os alunos tanto no ensino presencial como a distância. É importante destacar o fato de que nem tudo o que é ensinado pelo professor é aprendido pelo aluno, alguns buscam ajuda aos colegas, amigos e familiares, como apoio na aprendizagem.

E com o crescimento significativo nos últimos anos de alunos na modalidade à distância, tende a crescer e aumentar. Fato que ocorrem em todas as modalidades e que devem ter como objetivo principal a efetivação do processo de aprendizagem do educando e sua formação como um todo no que diz res- 
peito às competências cognitiva, social e afetiva (FERRAZ; BELHOT; SENO, 2005). Questões relevantes ao comportamento do aluno são normalmente alteradas de acordo com características inerentes ao ambiente e ao conteúdo com o qual o estudante interage (FERRAZ, 2008). Um mesmo aprendiz pode ter procedimento diferente quando muda de ambiente.

As preferências do aprendiz podem se modificar de acordo com o momento e assunto que ele está estudando. Delimitar o escopo de escolha poderá acarretar em possíveis falhas do processo de aprendizagem (ZAINA, 2008).

$O$ uso de técnicas de recomendação como suporte as metodologias de ensino adotado pelo professor, poderá corrigir estas falhas.

O presente estudo se norteia em primeiro momento em analisar as ações e as interações realizadas pelos alunos sobre os objetos disponibilizados dentro dos ambientes virtuais de aprendizagem e o desempenho da turma heterogênea, potencializado as correlações dos itens disponíveis no ambiente TelEduc e Moodle utilizadas no ensino de EAD, visando apresentar uma proposta de um sistema de alerta e recomendações em identificar o processo de mediação e interação no ambiente.

A seguir serão apresentados os trabalhos relacionados, métodos, cenário e experimentos e, por fim alguns resultados e o modelo proposto de recomendação.

\section{Trabalhos Relacionados}

Com o objetivo de entender e levantar os diversos problemas de heterogeneidade de turmas de níveis diferentes foi analisado algumas iniciativas e sistemas de recomendações para atender estas turmas.

Os trabalhos relacionados foram divididos em duas situações: (i) trabalhos com turmas heterogêneas (ii) trabalhos envolvendo siste- mas de recomendações.

Esta divisão tem como objetivo facilitar o entendimento sobre as diversas técnicas e soluções existentes para cada situação de implementação de ferramentas para AVAs baseadas em recomendação. Existem diversas propostas para agregar sistemas nos AVAs, e poucas para atender turmas de níveis de aprendizagem diferenciadas.

\subsection{Trabalhos relacionados Turmas Heterogêneas}

Natalie (2001) define as turmas heterogêneas como sendo compostas por alunos que diferem não só de forma multicultural, mas também em sua capacidade de aquisição de conhecimentos em suas habilidades. Podendo ser classificada em termos de idade, motivação, interesses, inteligência, conhecimentos das competências, atitudes e autodisciplina. A abordagem e a forma de trabalhar com turmas heterogêneas no ensino presencial não deve ser novidade para muitos professores, o problema está em como trabalhar no ensino a distância, com esta situação, sobretudo quando se trata de visualizar a conduta desses alunos no ambiente virtual de aprendizagem.

Segundo Schilling (2007), basta utilizar uma metodologia básica como ponto de partida para a aproximação a aprendizagem com turmas de diferentes níveis e de habilidades misturadas. Para ele o primeiro passo está na coordenação por parte do professor e, por parte do aluno, da diversidade e de sua capacidade em sala de aula. É preciso fazer uma análise das necessidades dos alunos e com as dados obtidas, os esforços para determinar como ajudá-los. Esta análise é importante para refletir sobre suas habilidades e estilos. E prepará-los para o fato de que a turma não será conduzida da forma tradicional, mas de forma colaborativa, todos no ambiente pode- 
rão auxiliar o aluno e aluno, aluno e professor, professor e aluno.

Se as turmas de ensino a distância forem maiores que as tradicionais em número de alunos, a atenção individual por parte do professor poderá não abranger a todos plenamente, os mais fracos não atingirão a compreensão da disciplina, logo, conhecerão a reação natural de evitar as atividades em que não são bem sucedidos (SCHILLING, 2007). Com as informações mapeadas das necessidades individuais dos alunos, o professor pode organizar os alunos em grupos funcionais de aprendizagem. A formação desses grupos será baseada nas habilidades e nos diferentes ritmos de aprendizagem. A construção destes permitirá o professor a rever a eficácia do seu plano de aula e que proporcione atribuições realistas e exequíveis para os alunos poder resolver. Os grupos poderão trabalhar juntos com base em seu perfil e nível de habilidade ou em tempo de o professor poder misturar os mais fracos com os mais avançados, fato que irá contribuir para a criação de método e variedade na turma. Este método permite que todos os alunos avancem em direção a um objetivo comum a um ritmo adequado para as suas capacidades e evita atrasar o seu ritmo a adiá-los com o material que estiver fora de sua aptidão.

Outro ponto relevante o autor Hofstede (2001, p9) definiu a cultura como "[...] a programação coletiva da mente de distinguir os membros de um grupo ou categoria de pessoas de outro [...]". A categoria pode se referir a países, regiões dentro ou entre nações, etnias, religiões, profissões, organizações ou entre os sexos. A definição mais simples é as regras não escritas do jogo social, que é possível identificar nas quatro dimensões como: o individualismo versus o coletivismo, fato que também ocorre em curso à distância é possível encontrar alunos que preferem trabalhar sozinhos; grandes ou pequenas distâncias, exemplo Polos de Ensino a Distância cidades que possui problemas de conexão a internet, forçando os alunos a deslocarem até o polo para resolver seus exercícios; isto forte versus fraco, alunos com domínio da tecnologia versus os alunos pertencentes à inclusão digital; incerteza versus certeza é o que provoca a evasão dos cursos, a incerteza da escolha; masculinidade versus feminilidade, nos cursos estudados não apareceu. Um fato bastante questionado são os efeitos das diferenças de linguagem entre professor e aluno, nos cursos de EAD, pode acontecer para cursos. Sendo o ônus da adaptação das situações transculturais de aprendizagem deve ser principalmente sobre os professores. Sanders (WRIGHT et al., 1997) realizou um estudo sobre o efeito do professor em sala de aula dentro do contexto do desempenho dos alunos para a avaliação de professores. O referido autor fez uma análise longitudinal do desempenho dos alunos e fez estimativas de escola, turmas, professores e outros possíveis efeitos. Ele analisou a magnitude relativa dos efeitos de professores sobre o desempenho do aluno ao mesmo tempo, considerando as influências da turma heterogênea, o nível de aproveitamento dos alunos e turmas no crescimento acadêmico. Sendo que os resultados mostram que os efeitos do professor são fatores dominantes e que afetam o ganho acadêmico dos alunos e as variáveis de contexto heterogeneidade entre os alunos e as turmas têm relativamente pouca influência sobre o ganho acadêmico. Assim, uma das principais conclusões é que os professores fazem a diferença e as implicações dos resultados de avaliação de professores e pesquisas futuras são discutidas.

Ben-Ari (BEN-ARI; KEDEM-FRIEDRICH, 2000) apresenta uma aplicação educativa que demonstra uma visão social construtivista, 
baseado nas teorias de Vigotsky $(1962,1978)$ e Piaget (1926) para atender uma turma heterogênea. Outro estudo foi também realizado por Faris (2009) para determinar quais os níveis de efeito foram associados e analisados na gestão de turmas heterogêneas, as características dessas, e também com as turmas homogêneas: o aumento da heterogeneidade dos alunos entre níveis acadêmicos, limites na medida em que os professores podem adaptar instrução com sucesso para satisfazer as necessidades individuais dos alunos; professores em turmas extremamente heterogêneas podem ser menos capazes de atender as necessidades individuais dos alunos; ela está associada a um menor grau de engajamento do aluno com o auxílio da tarefa; realização de menor capacidade de ganhos dos alunos tende a ser mais baixos em turmas heterogêneas; e os relatórios de observação das aulas indicam que os professores devem usar diversas estratégias para lidar com turmas extremamente heterogêneas, bem como a exigência de tempo dos professores e tutores e atenção, na gestão de suas aulas.

A prática nessas turmas heterogêneas redefine a ideia de que trabalhar com elas é um problema, na verdade esse tipo de trabalho é uma oportunidade, um desafio, pois permite que os melhores alunos se destaquem e possam estimular os demais. A heterogeneidade está presente no que diz respeito à forma como os estudantes entendem a disciplina com relação ao seu curso de formação, fato que é percebido em disciplina multidisciplinar, normalmente seletivas ou optativas. Outro exemplo está na abordagem de um assunto que agrada a todos os alunos, evento que ocorre em disciplina multidisciplinar, esta pode ser divida em dois tópicos: o primeiro apresenta artigos sobre novas tecnologias e linguagem de programação visual e multimídia, ou seja, mais próximo do grupo de alunos de engenharia e ciência da computação; o outro tópico trata de assuntos sobre pensamento e linguagem, neste caso o tópico fica mais próximo aos alunos com formação para os cursos de ciências humanas. O autor Mansetto (1994) destaca que o processo de ensinoaprendizagem permite ao aluno relacionar o que está aprendendo com os conhecimentos e experiências que já possui, permitindo também que o aluno entre em contato com situações concretas de sua vida fora da escola.

\subsection{Trabalhos Relacionados com Sistema de Recomendação}

Existem vários trabalhos relacionados com uso de sistemas de recomendação aplicados na internet, sendo considerado como uma rica área de pesquisa. Ele tem várias aplicações práticas e também definidas como sistemas que geram recomendação de pessoas (em Redes Sociais), como por exemplo, Orkut e Facebook. E também promove a recomendação de produtos e serviços (em e-training e e-commerce).

Os principais processos de um sistema de recomendação são mostrados na Figura 1 apresentado por Terveen (2001):

Alvo da recomendação: principal ator do processo e destinatário da recomendação. Ele pode apenas receber passivamente as sugestões ou pode fornecer também suas opiniões sobre os itens da base de informação - exemplo aluno e o professor/tutor.

Base de informação: onde se encontra todo o universo de escolhas e onde o alvo da recomendação deseja encontrar informação de seu interesse - exemplo ambiente de aprendizagem virtual (Moodle).

Provedor de recomendações: agente que seleciona e recomenda os itens da base de informação que têm maior relevância para alvo da recomendação. Para identificar quais 
são esses itens, o provedor pode se basear nas preferências informadas pelo próprio alvo da recomendação, nas preferências de outras pessoas e/ou no conteúdo dos itens - modulo de correlação.

Provedores de preferência: todo processo de recomendação está baseado em indicadores humanos de preferência. Assim, os provedores de preferência são tipicamente um conjunto de pessoas em cujas opiniões o provedor de recomendações pode se basear para fazer as sugestões - exemplo histórico dos alunos.

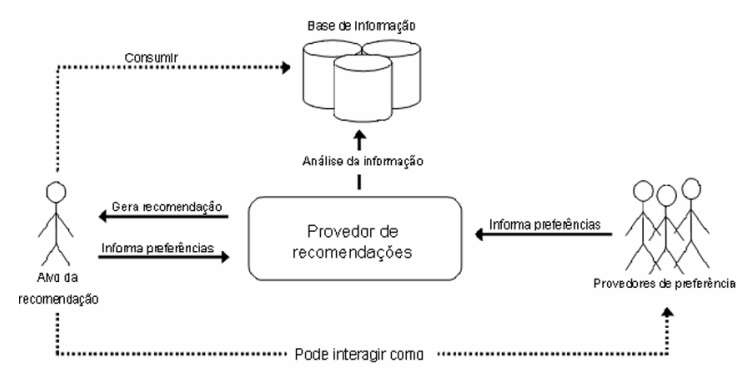

FIGURA 1 - Modelo do Processo de Recomendação FONTE: TERVEEN; HILL, 2001, p. 4.

Com base nestes processos, serão relatos a seguir alguns trabalhos com objetivo de auxiliar aprendizagem.

Olga Santos (2008) descreve em seu artigo um modelo para recomendações em cenários de aprendizagem, que foi concebido a partir de resultados empíricos por meio dos seguintes critérios de usabilidade e acessibilidade. Este modelo é compatível com os projetos de curso on-line em que descrevem as recomendações e apresenta informações adicionais para o usuário, com objetivo de explicar por que a recomendação foi enviada. O objetivo principal é melhorar a eficiência de aprendizagem. As informações utilizadas para o modelo do usuário foram: dados demográficos; afilia- ções (a instituição que o usuário pertence); preferências; línguas em que o usuário pode ler as informações; estilos de aprendizagem; nível tecnológico; nível de colaboração; nível de conhecimento por aprendizagem competência; nível de interesse e por aprendizagem de competências e acessibilidade (SANTOS, 2008). Estas informações foram usadas para selecionar as recomendações apropriadas para o usuário, quanto ao dispositivo e os recursos liberados. Utiliza um tamanho da tela para limitar o número de recomendações obtidas que se encaixam perfeitamente. Este tipo de sistema de recomendação permite ao professor um breve conhecimento do perfil do seu aluno, e com as informações poderão qualificar o primeiro encontro do professor com o grupo de alunos. No entanto, se Olga descreve recomendações em cenários, Fischer (FISCHER; WIVES, 2005) desenvolveu o protótipo de uma ferramenta, integrada ao Fórum de Discussão do Ambiente Virtual, apoiada em técnicas de recomendação de conteúdo, a fim de aperfeiçoar a utilização do fórum. Um dos problemas apontados pelo autor, em relação ao fórum de discussão, se refere à dificuldade do usuário em localizar as respostas às suas dúvidas, o que normalmente leva a criação de novos tópicos (perguntas), que podem ter sido criados anteriormente. Dessa forma, a ferramenta se propõe a "[...] indicar informações (mensagens) ao usuário descobrindo seus interesses através da análise do conteúdo das mensagens (perguntas e respostas) postadas no fórum [...]" (FISCHER; WIVES, 2005, p9.).

Em Nunes et al. (2006) é apresentado um Sistema de Recomendação para Apoio à Colaboração, suas recomendações são feitas a partir da análise do comportamento dos usuários em uma ferramenta Web Chat e do acesso a uma biblioteca digital. Apenas os dados cadastrais e o grau de interesse do usuário em um determinado assunto são considera- 
dos na recomendação. Os dados sobre o perfil do usuário são coletados de forma explícita.

Barcellos et al. (2007) apresenta um modelo de Sistema de Recomendação Acadêmico para Apoio a Aprendizagem, que dispensa a intervenção do usuário no momento de traçar seu perfil e utiliza técnicas de mineração de dados. O modelo foi implementado para verificar sua aplicabilidade. O protótipo Ponto com Filtro utiliza os dados que o usuário possui no seu currículo Lattes para realizar a recomendação. Uma das vantagens é que o usuário não precisa preencher formulários exaustivos para informar o seu perfil. O objetivo principal do sistema é fornecer ao usuário uma recomendação de artigos mais indicados ao seu perfil. A recomendação leva em consideração a área de interesse do usuário, seu nível de formação, áreas de interesse e idiomas. O perfil do usuário é criado de forma implícita, sendo as informações retiradas da sua página Web e do seu próprio currículo disponibilizado na plataforma Lattes.

Em Cazella et al. (2009), apresentam um modelo de sistema de recomendação de objetos de aprendizagem baseado em Filtragem Colaborativa e competências. $\mathrm{O}$ modelo permite que alunos recebam a recomendação de objetos de aprendizagem de forma automática conforme interesses do aluno, e de acordo com as competências que devem ser desenvolvidas dentro de um plano de aula. O protótipo implementado por autor permitiu recomendar conteúdo relevante para os alunos com o intuito de auxiliá-los no processo de desenvolvimento destas competências. Ferreira (FERREIRA; RAAB, 2010) desenvolveu um sistema de recomendação de objetos de aprendizagem no formato SCORM para o Ambiente Sophia/Moodle. O sistema se chama LorSys (Learning Object Recommender System) e as recomendações são baseadas nas informações contidas na tabela de preferência do estudante e utiliza duas técnicas: a filtragem baseada em conteúdo e a filtragem colaborativa. A Filtragem Baseada em Conteúdo será recomendada aos usuários objetos que possuem relação com o conteúdo do último objeto acessado pelo usuário. Para identificar as relações entre os objetos o sistema provê uma interface ao professor da disciplina, em que ele deverá definir o grau de relacionamento entre os objetos de aprendizagem que foram inseridos no MOODLE, classificando-os em: (i) Pré-Requisito; (ii) Complemento; (iii) Similar; e (iv) Sem relação.

Esse contexto tem sido trabalhado nos sistemas adaptáveis para aprendizagem, buscando focalizar nas inter-relações entre os usuários e o sistema. Nos cenários universitários, estudantes tendem a construir grupos para interação e realização de trabalhos extraclasse, esses grupos são criados, principalmente, baseados em empatia, similaridade e atração (NASS; LEE, 2000). Os estudantes nem mesmo consideram compatibilidade como um atributo sério. Eles estão muito mais interessados em parceria e amizade do que na eficiência de um grupo de trabalho. Dessa forma, estudantes universitários tendem muito mais a procurar parceiros com personalidades similares (como fariam em comunidades virtuais e Redes Sociais). Entretanto, o sistema que se julgar inteligente deverá ser capaz de detectar as necessidades dos usuários, de seguir os seus passos e finalmente adaptarem-se as suas necessidades e interesses de maneira colaborativa com seus usuários (TIFFANY; MCCALLA, 2003). Esse fato vem a certificar que, para o usuário dentro desse contexto, a sua opinião tem grande valor para sustentação desses sistemas, onde cada vez mais, segundo McSherry (2004), tem aumentando a consciência na pesquisa dos sistemas de recomendação, de fazer a necessidade do processo de recomendação mais transpa- 
rente aos usuários. A aplicação das técnicas de sistema de recomendação é uma das ferramentas que poderá apoiar o professor na visualização do seu ambiente de aprendizagem e dos perfis dos seus alunos. A partir da construção de uma base histórica desses perfis, com estes dados, o professor poderá adaptar os seus planos de ensino de acordo com as preferências dos seus alunos, fato que irá se ajustar ao longo de um período de análise de preferências. Um fato significativo dentro do ambiente de ensino é verificar com os seus alunos a importância de um determinado tópico no curso. Segundo Shardanand (SHARDANAND; MAES, 1995), a filtragem de informação social automatiza um processo de recomendação dito palavras de boca-a-boca, que poderá ser positivo ou negativo veja um exemplo. "Este curso é muito bom realmente fala da minha área de pesquisa" ou Eu não gostei do curso é muito técnico, tem muita programação, sendo que eu sou das ciências humanas. A diferença está presente no dia-adia dentro das salas de aula, o aluno em vez de questionar entre os seus colegas poderá verificar o grau de importância e de interesse dos alunos do semestre anterior e recomendar para os seus novos colegas alunos a importância dos tópicos e da disciplina. Esse histórico de interesses responderá muitas dúvidas que os alunos têm.

\section{Metodologia}

Foi utilizado, na pesquisa um estudo de caso (YIN, 2008), o método da observação direta, ou seja, exames planejados, deliberados e sistemáticos sobre o que está acontecendo, quem está envolvido e onde tudo estão acontecendo (LANKSHEAR, 2008). Com o propósito especificamente de coletar os dados registrados nos mecanismos de acessos ao ambiente
Moodle e TelEduc, utilizou-se da observação direta do tipo estruturado, que envolve planejamento e estrutura específica (LANKSHEAR, 2008), cujo objetivo foi avaliar a qualidade e a utilidade dos dados de acesso registrados no ambiente. Segundo Gil (2010), apesar de ser utilizado principalmente em pesquisa quantitativa, esse tipo de observação pode ser aplicado de forma bastante eficiente em métodos mistos. A observação direta estruturada deu-se através do AVA, onde ocorreu o processo investigado. No entanto, somente a observação não seria suficiente para uma validação do modelo, mesmo se tratando de uma pesquisa exploratória. Buscou-se então, nos procedimentos experimentais de natureza mais quantitativa, complementar a pesquisa. Entretanto, identificamos que não seria possível o controle da aplicação dos estímulos experimentais nem a distribuição aleatória dos sujeitos da pesquisa, características que, segundo Gil (2010) e Campbell e Stanley (1979), são indispensáveis para uma pesquisa experimental. Assim, elaboramos um pré-experimento de caráter exploratório para permitir a validação do modelo sem comprometer a presente pesquisa (CAMPBELL; STANLEY, 1979).

\subsection{Contexto da Pesquisa}

O perfil das turmas dos cursos de pós-graduação interdisciplinar ou multidisciplinar que inclui informática e educação é formado com alunos de diversas formações acadêmicas. Nesse contexto, foram observadas as ações dos alunos nos seguintes cursos: pós-graduação (lato sensu) em Informática na Educação (três turmas num total de 90 alunos), utilizando o TELEDUC, e MOODLE no curso em Informática instrumental (01 turma 137 alunos). Esses cursos possuíam alunos formados em: letras, biologia, pedagogia, matemática, química, psicologia, musica, informática, ve- 
terinária, física, engenharia, administração, entre outras, com percentual maior para as áreas humanas. E por este aspecto o maior número de alunos foi do sexo feminino.

Nos AVAs citados anteriormente, estavam disponíveis materiais preparados para atender a proposta do curso, como: agenda, apostilas on-line, recursos multimídia, vídeos, demonstrações e apresentações das disciplinas, além de slides impressos e CD-ROM com material para uso off-line. A primeira turma serviu como base para observação da conduta e do desenvolvimento do aluno no ambiente e análise das necessidades, como as dificuldades de acesso e no entendimento do conteúdo e nas atividades proposta pelo professor e tutor, participação nos fóruns e bate-papo, a observação de interação entre os colegas e o professor. Como estas observações foram possíveis verificar o que pode acontecer no ensino a distância com grupos de alunos heterogêneos. Para as demais turmas junto com o professor foram definida uma estratégia e metodologia para identificar o nível de conhecimento de cada aluno após as primeiras aulas do curso, bem como a formação de grupos.

\subsection{O Cenário da Pesquisa}

Nesta seção serão apresentados o cenário e as pessoas envolvidas e meio que ocorre o processo de aprendizagem. Os sujeitos da pesquisa foram os alunos, professor e tutor do curso de especialização em Informática na Educação. Esse curso foi escolhido por ser composto por professores universitários das áreas exatas e humanas e possibilitar a participação de alunos de diversas regiões no Estado do Rio Grande do Sul e em todo o Brasil. Dessa forma, foi possível identificar a turma heterogênea no curso, pelo fato de terem aluno e professor de diversas áreas do conhecimento. O ambiente virtual de aprendizagem foi o local a onde ocorreu todo o processo de ensino e aprendizagem, nele foi apresentado à disciplina, a todos os alunos no primeiro encontro presencial, bem como aplicação de um questionário que ajudou a colher subsídios para a validação de algumas hipóteses. Esse instrumento de coleta foi aproveitado do questionário do sistema de matricula na forma de um questionário estruturado. Com objetivo de obter informações do perfil do aluno. Estes dados foram exportados em XML junto com os relatórios de acesso ao ambiente, fórum, bate-papo, e-mail, atividades e material de apoio, maneira de identificar a presença ativa ou não.

Outro ponto importante foi à escolha da disciplina, sendo ela multidisciplinar, mesmo professor, ambiente, estratégia de ensino e conteúdo. A variação foi apenas no perfil dos alunos, no decorrer da análise e mudança de estratégia após a primeira observação e ajustes nas turmas seguintes.

Os procedimentos realizados de acompanhamento, observação e análise, ocorreram desde primeira aula presencial até a avaliação final. Utilizando essa etapa sem nenhuma intervenção. Somente analisando as necessidades do aluno e professor, através dos números de acessos no ambiente TelEduc e Moodle. A onde verificou alguns alunos que não tinham acessos registrados no ambiente. Nesta situação foi consultada a secretaria do curso, para confirmação dos nomes de alunos que cancelaram a matrícula. Estes casos, para os quais não obtiveram nenhum acesso ao ambiente foram considerados como perdas na observação.

A característica da turma foi observada no desenvolvimento de atividade multidisciplinar, composta por alunos de diversas regiões do Estado e Nacional e de diferentes níveis de conhecimentos, mas com uma base de conhecimento já construída em suas áreas 
afins, alunos apropriados ao curso de especialização.

Na observação foi possível verificar como aconteceu a necessidade do aluno e o porquê aconteceu. Ao deparar com uma atividade típica para alunos de informática, ocorreu a dificuldade na execução, bem como busca do material de apoio e suporte de colegas, os acessos se destacaram no ambiente, principalmente nos itens mais relevantes do ambiente como: fórum de dúvidas, e-mails, material de apoio, chat e atividades, e visita aos portfolios dos colegas. No resultado final da observação, deparamos com duas situações: os alunos com dificuldades e os que não possuem, mas não cooperam.

\subsection{Material e Métodos}

Em busca de soluções para atender as necessidades dos alunos e professores, foi construída uma planilha como um instrumento que continha os dados biográficos e foram incluídas informações dos formulários de inscrições solicitando idade, sexo, formação escolar, conhecimentos informáticas e educação à distância. Além disso, foi coletado do questionário o tempo e ocupação, como outros cursos online, carga horária de trabalho e tipo de escola (particular ou municipal ou estadual). Com a preocupação nos critérios de elegibilidade, foram incluídos no estudo todos os alunos, e não foram excluídos do estudo, aqueles casos de matrículas de alunos especiais, uma vez que objetivo era analisar o comportamento de todos os alunos no ambiente virtual de aprendizagem. E, portanto tentar assim identificar pontos e fatores associados ao processo de interação, mediação e trocas sociais, independente da avaliação parcial e final do curso, mas que poderiam servir como balizador para uma próxima análise de qualidade do processo de aprendiza- gem. Não foram considerados significantes os acessos para o estudo os alunos que apresentaram pouca interação no ambiente e, por esta decorrência efetuaram o cancelamento. Os pontos importantes desta observação focaram no questionamento e avaliação do comportamento dos alunos nos acessos das ferramentas disponíveis para seu uso no ambiente, a qual se destaca: material de apoio, fórum, atividades, bate-papo e e-mail, wiki. Estes dados foram coletados dos relatórios do TelEduc e Moodle e nos formulários de inscrições e matriculas dos alunos. Estando equipados com estas informações em planilhas, permitiu ao professor verificar o perfil heterogêneo da turma, destacando entre elas a formação acadêmica dos alunos, com alunos tanto das áreas exatas e humanas.

Para realização do experimento foi escoIhida uma turma com perfil heterogêneo com uma média de 30 alunos com desenvolvimento de tarefa multidisciplinar, compostas por alunos diversas formações acadêmicas e de diferentes níveis de conhecimentos, mas com uma base de conhecimento já construída em suas áreas afins, alunos apropriados ao curso de especialização.

O método utilizado para atender as necessidades do professor e aluno, foi os resultados da analise do questionário.

Com os resultados do perfil do aluno possibilitou ao professor identificar o tipo de turma heterogênea ou homogênea e adotar novas estratégias para atender as observações realizadas na primeira turma.

Apesar disso foi construindo com o professor uma proposta de identificar a zona proximal dos alunos dentro do período do curso. Mas para isso adotou-se aplicação de atividades de grau fácil, médio e avançado, como instrumento de medição para verificar os estágios de aprendizagem e a evolução dos mesmos da zona desenvolvimento real (ZDR) 
passando pelos estágios da zona de desenvolvimento proximal (ZDP) até a nova ZDR, baseado nos estudos de Vigotsky (1998). Evitou-se de aplicar um questionário de sondagem do seu pré-conhecimento na disciplina, mas procurou iniciar a disciplina com conceitos básicos para aproximar a todos no conteúdo proposto pelo professor, e após esta revisão de conceitos aplicou-se uma atividade de nível fácil, para identificar o seu grau de conhecimento. Utilizou-se o experimento em dois momentos, no primeiro momento aplicou o estudo no ambiente TELEDUC e no segundo momento no ambiente MOODLE.

\subsubsection{Experimento ambiente TELEDUC}

Este experimento começou com os objetivos do plano ou programa de ensino da disciplina, em busca de obter mais informações sobre os objetivos, a metodologia proposta e o desempenho das turmas anteriores a partir da combinação de diversos critérios e da adoção de uma perspectiva que focaliza não somente resultados, mas o ciclo de aprendizagem do aluno, conforme seu perfil, principalmente em turmas heterogêneas. Questionado o professor, sobre atenção aos alunos heterogêneos, verificou-se que não tinha esta diferenciação, pois atenção era para todos. O experimento foi realizado com três turmas após as observações realizadas com a primeira e constataram-se duas necessidades importantes por parte do professor e tutor:

- Disponibilizar aos alunos no começo do semestre a proposta clara da disciplina;

- procurar registrar comentários em todas as atividades realizadas por eles nos seus portfólios e fóruns, principalmente para os alunos participantes ativos no ambiente, de forma motivadora, fato observado nos e-mails dos alunos enviados para os tutores da disciplina.

No entanto os alunos menos habituados ao ambiente de ensino a distância e principalmente nas realizações de atividades práticas, necessitaram de acompanhamento mais próximo, como explicações adicionais ao entendimento do conteúdo em etapas, como exemplo: estar disponível no ambiente exemplo prático para que os alunos tentem compreender e adaptar em todas as atividades (exemplos, de trabalhos já realizados por alunos anteriores). Nesta situação, é útil a construção de uma ferramenta de apoio ao professor de forma automática ou semiautomática de sugerir exemplos práticos ou mostrar os passos dos alunos que obtiveram sucesso nestas etapas, e informação sobre a etapa em que cada aluno se situa em forma de alertas ou recomendações. Outro fato observado é que muitos ficam omissos nas participações de bate-papo ou realizações das tarefas, deixando tudo para o final. O motivo, o não entendimento claro da atividade a ser executada. $\mathrm{Na}$ sequência do processo de observação e acompanhamento, surgiram algumas dificuldades de entendimento entre alunos e professor no esclarecimento de dúvidas através da ferramenta bate papo disponível pelo ambiente TelEduc. O bate papo é uma ferramenta importante para o professor, pois é canal de comunicação on-line, permitindo ao aluno a interação com o todo, podendo surgir o momento de AJUDA entre os colegas no entendimento do conteúdo. Esta ferramenta possibilitou ao professor e ao aluno rever as dúvidas pontuais o que permitiu a ambos uma análise posterior.

Desta forma podemos considerar útil a construção de ferramentas de alertas, que permitem criar formas de registrar as ações efetuadas pelos alunos durante as atividades, para posterior reprodução, possibilitando aos 
docentes observar o percurso seguido pelos alunos e assim ter uma melhor percepção das dificuldades enfrentadas e atuar em conformidade. Os dados foram analisados quantitativamente através de técnicas estatísticas descritivas de medidas repetitivas, correlações, moda e mediana. A correlação entre as duas principais variáveis do ambiente de EAD, conteúdo e atividades, ou seja, o aluno que tiver alguma dificuldade em resolver as atividades, poderá também ter dificuldade em entender o Conteúdo disponível no Material de Apoio, sendo que o item que mais depende deste é o item Atividades. Portanto é possível identificar esta situação pelos seus acessos, que para valores altos em Atividades (X) teremos associados valores altos em Material de Apoio $(Y)$ e para valores baixos em $X$ valores baixos em $Y$ aqui define como correlação direta. Porém cabe também registrar que este experimento foi submetido à avaliação pelo departamento estatístico da UFRGS e foram realizadas em duas etapas distintas, para validação das correlações de acessos as ferramentas disponíveis no ambiente. Utilizou-se o software estatístico SPSS (Statistical Package for Social Science).

\subsubsection{Experimento ambiente MOODLE}

Para atender a proposta do professor e identificar a viabilidade de modelar um sistema de recomendação, aproveitou a disciplina para simular o ambiente de alertas e recomendação para verificar se esta solução pudesse ajudar os alunos heterogêneos e que fosse possível avaliar e validar a construção do protótipo. Então procurou simular duas etapas:

A primeira etapa: foi liberado aos alunos em familiarizar com o material da disciplina, possibilitando o mesmo a contribuir na cons- trução do material, disponibilizado em WIKI e no fórum discussão sobre o texto, para que o mesmo possa recomendar e contribuir com novos textos. A cada participação do aluno disparou mensagens de alertas e recomendações enviadas por e-mail, tanto para os alunos e professor.

A primeira mensagem enviada foi pela participação dos alunos no fórum, conforme mostra na Figura 2. O texto e a resposta tiveram como objetivo provocar alertas para o professor e recomendações para os alunos. O questionamento foi se os alunos recomendariam o primeiro texto, que foi disponibilizado pelo professor no ambiente.

Esta mensagem foi acionada após a contribuição do primeiro aluno no fórum, com objetivo de motivar e agradecer a contribuição no ambiente e solicitar recomendação por parte do aluno.

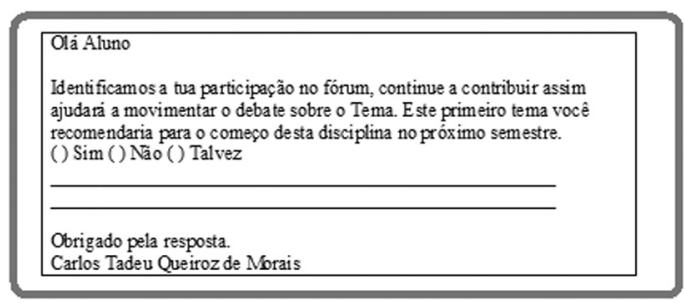

FONTE: produzido pelo autor (2011)

Em seguida recebem-se alguns e-mails, conforme mostra a Figura 3, que também procurou preservar a identidade do aluno, mas respeitamos a data e hora do envio da resposta para certificar a origem e a veracidade das informações.

Nota-se no texto da mensagem a necessidade e a importância da contribuição dos colegas nos fóruns. A próxima mensagem veio a contribuir, com o professor conforme o texto da mensagem na Figura 4, que talvez a demora em postar, esta no entendimento do mate- 


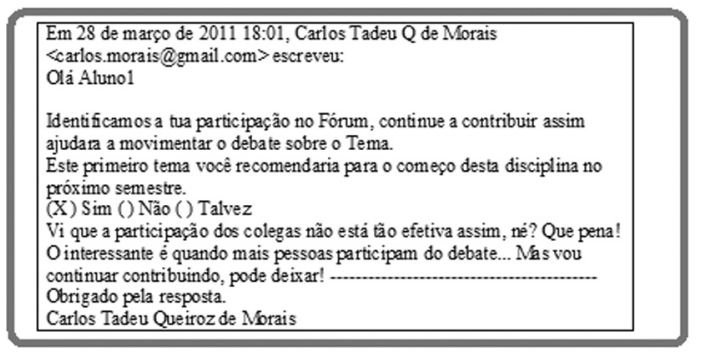

FIGURA 3 - Mensagem Recomenda-1 FONTE: produzido pelo autor (2011)

rial disponível, para este perfil de aluno, que neste caso ele recomenda ao professor, que seja trocado os textos, para que então tenha uma melhora na postagem.

\begin{tabular}{|l|}
\hline Em 29 de março de 2011 12:12, Carlos Tadeu Q de Morais \\
<carlos.morais@gmail.com> escreveu: \\
Olia Aluno2 \\
Identificamos a tua participação no Fórum, continue a contribuir assim ajudara a \\
movimentar o de bate sobre o Tema. Este primeiro tema você recomendaria para o \\
começo desta disciplina no próximo semestre. \\
\\
() Sim (X) Nào () Talvez \\
Não, porque eu tive dificuldade para escrever um tex to meu sobre EAD. Eu \\
entendo que o tex to dois deveria ser antes do texto um, pelo menos, as meus olhos, \\
pois foi essa a minha dificuldade em postar a minha primeira contribui cão. Logo \\
recomendaria que o texto 1 fosse o segundo. ok? Sempre que vc precisar da minha \\
ajuda, contribuição estou a disposiçâo. ok? \\
Abração alegre, Aluno2 \\
Obrigado pela resposta. \\
Carlos Tadeu Queiroz de Morais
\end{tabular}

FIGURA 4 - Mensagem Recomenda-2 FONTE: produzido pelo autor (2011)

Ao localizar a baixa participação nos fóruns disparou um alerta para os alunos, com o seguinte texto, conforme mostra a Figura 5 , provocando aos mesmos a participar, bem como referenciando os alunos 1,2 e 3, pela participação e destacando a importância das contribuições.

Aproximadamente 14 horas depois o Alerta II, provocou um retorno de um aluno que estava com baixa participação no ambiente, conforme mostra a Figura 6.

Com objetivo de aumentar a participação o professor disponibilizou um novo fórum que em seguida os mesmos alunos que iniciaram a participação no fórum I, também foram

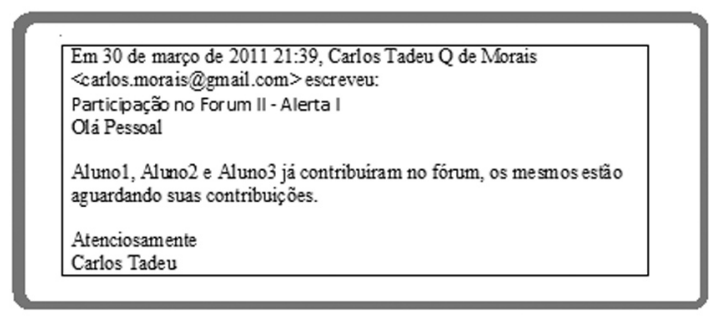

FIGURA 5 - Mensagem Alerta I FONTE: produzido pelo autor (2011)

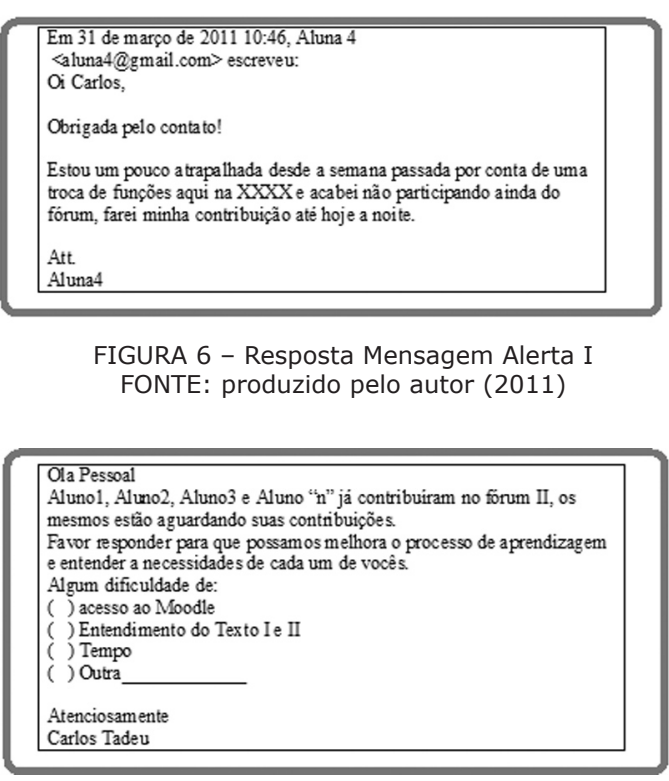

FIGURA 7 - Mensagem Alerta II FONTE: produzido pelo autor (2011)

pontuais na participação deste novo. Após 24 horas disparou um novo alerta em função do fórum II. Neste novo alerta teve com objetivo de verificar a dificuldade dos alunos em participar no ambiente e contribuir nos fóruns, acrescentando novas alternativas, como mostra na Figura 7, mensagem de envio, para que o aluno possa dizer a sua necessidade, pois muitas vezes fica oculta para os professores e tutores.

A resposta foi unânime a onde destacou a alternativa "tempo" conforme mostra a Figura 8 a seguir. 


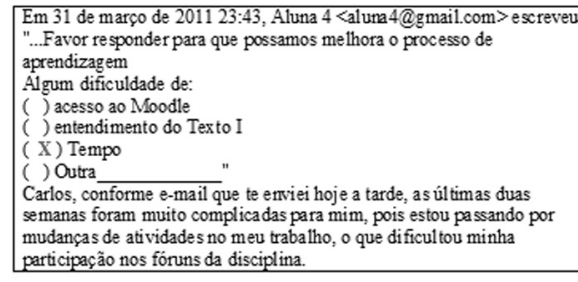

FIGURA 8 - Resposta Mensagem Alerta II FONTE: produzido pelo autor (2011)

Esta variável "tempo" é possível mensurar a ocupação dos alunos com as atividades externa ao curso, conforme os dados que consta no questionário aplicado no primeiro dia do curso.

Segunda etapa, como trabalhar com alunos de formações diferentes, e como nivelar o seu conhecimento e aproximar sua zona desenvolvimento proximais dentro da disciplina em vigor? A resposta a esse questionamento buscou-se com as atividades elaboradas junto com o professor, a onde foram construídas três atividades/estágios e aplicadas em momentos distintos.

O primeiro estágio classificou com atividade de nível fácil, para que todos independente de formação, mas que pudesse executá-la. Após o resultado atribuído pelo professor no ambiente, com o parecer OK ou NOK.

O segundo estágio selecionou-se em planilha pelo perfil do aluno a construção de um grupo heterogêneo composto por nível de conhecimento e formação diferenciada, para provocar a troca de conhecimento entre si. Portanto para isso foi disparado uma segunda tarefa, classificada de nível médio para que ambos pudessem buscar o conhecimento juntos.

O terceiro estágio teve como objetivo de ampliar o grupo a consolidação do aprendizado, definida na atividade classificada de nível difícil, tendo assim a necessidade da cooperação de todos para execução da tarefa.
No final se pode verificar período de aproximação e crescimentos dos alunos conforme mostra os estágios.

\section{Resultados e Discussão}

Através do estudo de observação, esta pesquisa empírica com abordagem quantitativa, procurou avaliar as relações existentes entre os objetos que estão inseridos dentro de um ambiente de ensino virtual, como neste caso a ferramenta TelEduc os itens: material de apoio; atividades; bate papo e e-mail. O item material de apoio representa o repositório de textos, vídeos, apresentações e artigos para fundamentar os tópicos da disciplina, inseridos pelo professor. Pela análise de regressão múltipla buscou-se conhecer o quanto o material de apoio, o e-mail e bate-papo (variáveis independentes) influenciam a atividade (variável dependente). A análise dos resultados foi construída com base nos dados de acesso e alguns questionários disponíveis diretamente no AVA Moodle e TelEduc, bem como observações no ambiente, foram necessários à visualização individual das respostas de cada um dos alunos para tabular e avaliar os resultados de forma estatisticamente. Isso porque os AVAs não possuem um módulo de relatórios que fizesse os cruzamentos dos dados e a correlação. O levantamento de conceitos, modelos e instrumentos de análise de correlação contribuíram para o entendimento do problema em foco e para a proposta de solução e implementação de melhoria nos AVAs.

Através da técnica de filtragem colaborativa (HERLOCKER et al., 2011) é possível achar a similaridade de acessos no ambiente, conforme o algoritmo a seguir:

Para cada item AVA, I (item), Para cada aluno A que acessou I1, 
Para cada item I2 acessado pelo aluno A

Registra que o A acessou I1 e I2

Para cada item I2

Calcula a similaridade entre I1 e I2

Calcular a similaridade (BADRUL et al., 2001) entre os alunos permite encontrar um conjunto de alunos mais próximos com sua conduta dentro do ambiente, os históricos dos acessos da primeira turma serviram com base de recomendação para turma seguinte. Outro ponto relevante foi para os itens não acessados pela análise dos vizinhos mais próximos. Ao usar essa abordagem proporcionou entender a conduta e a aprendizagem dos alunos e turmas heterogêneas, em três momentos distintos.

No primeiro foram as observações e anotações dos históricos de cada aluno de diferentes perfis, para que então pudesse comparar com a segunda turma e identificar suas ações e reações no ambiente, através da similaridade dos acessos no AVA, se os interesses do aluno $X$, são similares aos alunos $Y$, os itens preferidos de acessos pelo aluno $Y$, podem ser recomendados ao aluno $X$, de acordo com seu perfil e produção das suas tarefas. Avaliação de similaridade entre alunos pode ser feita pelo histórico de acesso as ferramentas ou pelos itens associados, ou seja, pela correção item a item (SANTOS; BOTICARIO, 2009; MCSHERRY, 2004). Com resultado da correlação, utilizou na terceira a opção de trabalhos em dupla. A tarefa com qual o sujeito se defronta no contexto experimental com algo novo, além de seu conhecimento real, baseado na sua formação superior, e não pode ser resolvida com as habilidades que já possui. Nesse caso, foi colocada no ambiente uma tarefa nova com uso de uma linguagem de multimídia desconhecida pelos discentes e frequentemente foi possível observar a reação dos alunos no ambiente, com o estímulo neutro e incluído na situação de tentar resolver um problema. Apesar de oferecer um estímulo simples dos quais se esperava uma resposta imediata do comportamento dos alunos. Durante a observação foi nítida a necessidade da ajuda do outro, principalmente em grupos de alunos mais próximos a conhecimento da tarefa. Contudo ao surgir à necessidade do entender o processo de desenvolvimento do aluno, a interação e a mediação por parte do professor e tutor, este estudo procurou priorizar a análise de fatores relevantes no ambiente virtual de aprendizagem em nosso meio, como professor e aluno, para que pudéssemos dar uma maior contribuição no sentido de intervenção, porém várias hipóteses não puderam ser testadas, por não termos obtido as informações necessárias nos perfis dos alunos, já destacadas. Apesar de haver alguns relatos, de que alguns alunos, que chegaram a utilizar os AVAs e não consegue de imediato familiarizar com os módulos disponíveis, pois muitas vezes esta estrutura básica são fatores preditivos para realização das atividades. No entanto foi possível visualizar a conduta de alguns alunos, constatou-se que os mesmos somente entravam no ambiente, mas logo saíam e mais tarde retornavam, e na sequência dos seus logs observou-se, a entrada no ambiente, agenda, logoff, ao retornar a entrada no ambiente, agenda, material de apoio, logoff, novamente um novo acesso, entrada no ambiente, material de apoio, atividade e logoff, tudo no mesmo dia. Ou seja, deduz a busca da familiarização com o ambiente. Para que se possa entender este processo na Figura 9 (THARP; GALLIMORE, 1988), busca-se exemplificar este momento, a onde um sujeito que busca o conhecimento através de tentativa e erro, e logo após busca ajuda dos colegas e tutores, baseou-se nos quatros estágio da ZDP. 


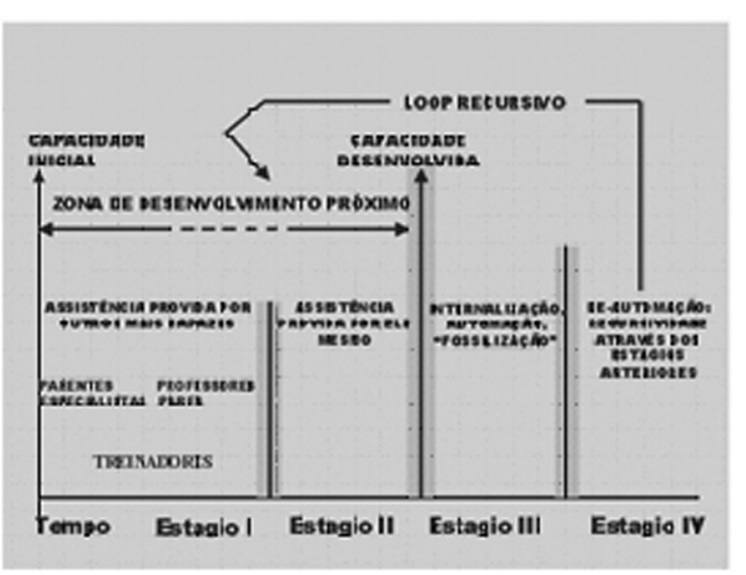

FIGURA 9 - Mensagem Alerta I

FONTE: Tharp e Gallimore (1988, p. 35)

Aplicação dos estágios de nivelamento foi o melhor resultado para este estudo, pois com ele, validou a metodologia de aplicação de tarefas de nivelamento do conhecimento dos alunos de forma individual e coletiva, atendendo os resultados esperados pelo professor, como é visto na Figura 9, em paralelo a Figura 8, o estagio IV ficou visível na apresentação do trabaIho final da disciplina, a onde foi contemplada a participação de todos no resultado individual de cada aluno. Conhecer a influência desses estágios é importante para a etapa de recomendar ao professor, que agregada à análise de outros fatores, que possibilita adequação de novas estratégias e metodologia de um bom plano de ensino, instrumento vital para o curso. Com a identificação foi possível ajustá-los mediante algumas recomendações de forma manual. A amostragem pareceu coerente com os conceitos da ZDP, pois observou-se que os alunos na fase inicial de sua aprendizagem necessitam de mais apoio do tutor, de um acompanhamento mais detalhado para desencadear ações mais direcionadas.

\subsection{Modelo de Recomendação}

O modelo proposto de recomendação é composto basicamente pelos seguintes módulos:

Módulo perfil dos usuários, os atores deste módulo são principalmente os alunos e os professores formados pelas disciplinas do curso, cadastrado no ambiente TelEduc ou Moodle. Este módulo tem como função obter informações como a formação acadêmica, nome, cidade e Estados são fixas por disciplina, e que poderão ser definidos explicitamente pelo o perfil para a filtragem e recomendação.

Módulo de Correlação para obter informações. Os dados de entrada do sistema são considerados não estruturados, e gerados continuamente integrados pelos ambientes virtuais de aprendizagem através de um API. E que será necessário definir padrões na criação dos textos para geração de correlações presentes nas variáveis e eventos do AVA de modo que possa aumentar a eficiência do processo de filtragem. Os dados serão disponibilizados em formatos $X M L$, que será necessário então desenvolver um submódulo para capturar as informações provenientes do AVA.

Módulo para Recomendação, sistema para filtragem das informações realizando recomendações baseadas em conteúdo. A partir das informações adquiridas pelo sistema anterior e do perfil dos usuários do sistema inicial, será realizado a filtragem dessas informações, identificando os pontos críticos e relevantes para esses usuários. Para recomendar as ações pertinentes ao professor será utilizada uma arvores de decisão, que em conjunto com a técnica de estatística irão armazenar o conhecimento do sistema e complementar o processo de recomendação das ações. O Modelo da Aplicação de Recomendação, os dados de entrada do sistema são as transações efetuadas pelos alunos de um site de AVA, neste caso especifica o TelEduc ou Moodle em uma perspectiva histórica 
do período letivo de cada turma. Estes dados são utilizados para montar a matriz alunoevento. Questões como a quantidade de itens e a frequência de acesso dos itens que estão incluídas no ambiente. Em relação ao professor, para o qual se deseja fornecer recomendações em tempo real, são observadas, dados dos alunos com pouca freqüência informações sobre seu histórico, as informações contidas nos acessos de consultas as ferramentas durante a interação com o site. A saída do modelo de sistema consiste em uma lista das $n$ melhores recomendações para um professor, onde $\mathrm{n}$ é um dos parâmetros a ser configurado no sistema. Estes itens selecionados serão utilizados pela aplicação do sistema, que determina o que apresentar ao professor e de que maneira ou em que contexto.

O método de recomendação, componente central de um sistema de recomendação, constitui o verdadeiro escopo deste trabalho. Ele será desenvolvido tendo como base os dois métodos de sistemas de recomendação mais conhecidos:

- correlação item-a-item: permite prover ações personalizadas de acordo com o que o aluno possui em sua escolha de itens de ferramentas no ambiente;

- correlação pessoa-a-pessoa: dá um caráter mais individualizado ao fazer uso das informações históricas do aluno para produzir sugestões.

De acordo com a taxonomia que está sendo analisada e como consequência da aplicação do método de recomendação híbrido (BURKE, 2002) constata-se que o grau de personalização do sistema é persistente, já que, além de informações da sessão - módulos do ambiente -, também serão utilizadas informações do histórico dos acessos dos alunos. A forma de apresentação das recomendações fica por conta da aplicação do sistema proposto. Pode ser passiva, ao expor os itens durante a navegação pelo site, ou baseada em tecnologia de extração em formato XML, possibilitando importar para outra base de dados ou diretamente no Site. O processo de geração de recomendações pode ser dividido em três partes: representação; construção do modelo e geração das recomendações. A primeira parte, a representação, trata do esquema utilizado para modelar os itens do ambiente virtual de aprendizagem que já foram acessados pelo aluno, bem como os itens que eventualmente estejam acessando durante o curso que não constam como prioridade para análise.

A segunda parte a construção do modelo diz respeito à tarefa de determinar de formas numéricas e estatísticas das relações entre aluno e entre itens e organizar estas informações de forma a deixá-las facilmente acessíveis para a próxima fase.

A terceira e última parte, geração das ações de recomendações, trata do problema de identificar os itens que mais recomendáveis para um aluno a partir das informações de análise de correlações e similaridade, e de seus itens mais ou menos acessados.

A Geração das Recomendações - esta é a etapa final de todo o processo do sistema de recomendação e na qual o modelo definitivamente combina as informações processadas pelas técnicas de correlação item-a-item e filtragem colaborativa. Durante a navegação dos alunos pelo site do AVA, o conjunto U de itens inseridos no registro de acessos de cada aluno é utilizado para gerar os tops- $\mathrm{N}$ recomendações, da seguinte maneira: $O$ conjunto $\mathrm{C}$ de itens disponíveis para novo aluno é obtidos através da recuperação dos $\mathrm{m}$ meIhores itens gerados pela filtragem colaborativa e armazenados no banco de dados. Os itens que já estejam em $U$ são removidos de C. Para cada c $\square$ C, é calculada a sua similaridade com o conjunto $U$, como a soma das 
similaridades entre todos os itens $\mathrm{j} \square \mathrm{U}$ e c. $\mathrm{O}$ valor da similaridade entre c e $\mathrm{U}$ é ponderado pelo valor correspondente de c gerados no processo de obtenção dos $m$ melhores itens na filtragem colaborativa. Por fim, o conjunto $C$ é ordenado em ordem decrescente de similaridade e os primeiros $\mathrm{N}$ itens são selecionados como o conjunto das $\mathrm{N}$ melhores recomendações.

\section{Conclusão}

Certamente existem outras variáveis não inclusas nesta avaliação - variáveis perturbadoras - relacionadas com o nível de conhecimento dos alunos, que provocam alterações nas duas variáveis e que estamos a estudar e que explicam a forte correlação analisada. Sob as condições deste experimento, frente aos resultados obtidos, parece licito concluir que: É muito complicado aplicar uma metodologia que respeitasse o principio de um sistema de recomendação sem automatização do processo, mas que, ao mesmo tempo, pudessem surgir efeitos nas ações e reações dos alunos, mesmo se não aderisse a ele completamente, afinal se tinha que discorrer as técnicas de comportamentos similares. O maior problema era resolver a integração entre os dados informativos e históricos de cada ferramenta, afinal apresentar um estudo sobre este enfoque, exigia um mínimo de contextualização histórica do todo. Entretanto, a diferença seria percebida nos resultados da análise estatística aplicada a cada item. A intenção, contudo, não é conduzir a pesquisa nesta análise, mas produzir parâmetros de um dispositivo deflagrador das informações embutidas nos históricos dos alunos suas ações e reações, isto é, possibilitando soluções de ajudar o aluno e professor a identificar seus gargalos, suas dificuldades, motivando ao aluno encontrar seu próprio caminho com o sem o professor. Contudo pretende-se utilizar os conceitos de ZDP, com objetivo de propor um sinalizador que permite mostra dentro do ambiente virtual de aprendizagem situação de alertas de aprendizagem dentro do ambiente.

\section{Agradecimentos}

Nossos agradecimentos à CAPES (Coordenação de Aperfeiçoamento de Pessoal de Nível Superior), Projeto STICAmsud LWM.

\section{Referências}

BADRUL, S. et al. Item-based collaborative filtering recommendation algorithms. In: INTERNATIONAL CONFERENCE ON WORLD WIDE WEB, 10., 2001, Hong Kong. Proceedings Hong Kong: [s.n.], 2001. P. 285-295.

BARCELLOS, C.D. et al. Sistema de Recomendação Para Apoio a Aprendizagem. RENOTE: Revista Novas Tecnologias na Educação, Porto Alegre, v. 5, n. 2, 2007.

BASSANI, P.B.S. Mapeamento das Interações em Ambiente Virtual de Aprendizagem: uma possibilidade para avaliação em educação a distância. 2006. Tese (Doutorado) - Programa de Pós-Graduação em Informática na Educação, Universidade Federal do Rio Grande do Sul, 2006, Porto Alegre, BR-RS. 
BEN-ARI, R.; KEDEM-FRIEDRICH, P. Restructuring heterogeneous classes for cognitive development: Social interactive perspective. Instructional Science, Amsterdam, v. 28, no. 2, p. 153-167, 2000.

BURKE, R. Hybrid recommender systems: Survey and experiments. User Modeling and User-Adapted Interaction, Dordrecht, v. 12, p. 4, p. 331-370, 2002.

CAMPBELL, D.T.; STANLEY, J.C. Delineamentos Experimentais e Quase-Experimentais de Pesquisa. São Paulo: EDUSP, 1979.

CAZELLA, S.C. et al. Recomendação de Objetos de Aprendizagem Empregando Filtragem Colaborativa e Competências. Anais do Simpósio Brasileiro de Informática na Educação, Florianópolis, ano 20, 2009. Disponível em: http://www.br-ie.org/pub/index.php/sbie/article/view/1158/1061 Acesso em: Acesso em: 20 jul. 2011.

FARIS, A.O. The Impact of Homogeneous vs. Heterogeneous Collaborative Learning Groups in Multicultural Classes on the Achievement and Attitudes of Nine Graders towards Learning Science. [S.I.]: ERIC Education Resources Information Center, 2009. ED504109.

FELDER, R. Matters of Style. ASEE Prism, Washington, v. 6, no. 4, p. 18-23, dec. 1996.

FELDER, R.; SILVERMAN, M.; LINDA, K. Learning and Teaching Styles in Engineering Education. Engineering Education, v. 78, no. 7, p. 674-681, 1988. Disponível em: <http://www4.ncsu.edu/unity/lockers/users/f/felder/public/Papers/LS-1988.pdf> Acesso em: 20 jul. 2011.

FERRAZ, A.P. do C.M. Instrumento Para Planejamento de Materiais Instrucionais Para Educação a Distância. Tese (Doutorado) - Escola de Engenharia de São Carlos, Universidade de São Paulo, 2008, São Carlos, BR-SP.

FERRAZ, A.P. do C.M.; BELHOT, R.V; SENO, W.P. Educação à Distância: diretrizes e contribuições para a implantação dessa modalidade em instituições educacionais. Colabor@: Revista Digital da CVA-REICESU, Curitiba, v. 3, n. 9, jul. 2005.

FERREIRA, V.H.; RAAB, A.L.A. LORSys: um Sistema de Recomendação de Objetos de Aprendizagem: SCORM CINTED-UFRGS. RENOTE: Novas Tecnologias na Educação, Porto Alegre, v. 8, n. 2, jul. 2010.

FISCHER, C.G.; WIVES, L.K. Recomendação de Conteúdo em Fóruns Eletrônicos. In: III FÓRUM DE INTELIGÊNCIA ARTIFICIAL, 3., 2005, Canoas. Anais. Canoas: ULBRA, 2005. p. 9 - 15.

GIL, A.C. Como Elaborar Projetos de Pesquisa. 5. ed. São Paulo: Atlas, 2010.

HERLOCKER, J. Understanding and Improving Automated Collaborative Filtering Systems. 2000. Tese (Doutorado) - Faculty of The Graduate School, University of Minnesota, 2000, Minnesota, USA. Disponível em: <http://web.engr.oregonstate.edu/ herlock/papers/thesis.pdf> Acesso em: 12 maio 2011. 
HERLOCKER, J. et al. Evaluating Collaborative Filtering Recommender Systems. ACM Transactions on Information Systems, New York, v. 22, no. 1, jan. 2004. Disponível em: <http://web.engr.oregonstate. edu/ herlock/papers/eval_tois.pdf> Acesso em: 12 maio 2011.

HOFSTEDE, Geert. Culture's Consequences: Comparing Values, Behaviors, Institutions and Organizations Across Nations, 2nd Edition. 596 pages. Thousand Oaks CA: Sage Publications, 2001.

KARPOV, Y. Vygotsky' doctrine of scientific concepts: Its role for contemporary education. In: KOZULIN, A.; GINDIS, B.; AGEYEV, V.; MILLER, S. (Ed.). Vygotsky's educational theory in cultural context. Cambridge: Cambridge University Press, 2003. P. 138-155.

LANKSHEAR, C.; KNOBEL, M. Pesquisa Pedagógica: do projeto à implementação. Porto Alegre: Artmed, 2008.

LOPES, G.R.; SOUTO, M.A.; WIVES, L.K.; OLIVEIRA, J.P.M. de. A Personalized Recommender System for Digital Libraries. In: SIMPÓSIO BRASILEIRO DE SISTEMAS MULTIMÍDIA E WEB, 14., 2008, Vila Velha. Anais do WebMedia. Porto Alegre: SBC, 2008. P. 59-66.

MCSHERRY, D. Explanation in recommender systems. In: ECCBR 2004 WORKSHOPS, 2004, Madrid. Proceedings. Madrid: Universidad Complutense de Madrid, Departamento de Sistemas Informáticos y Programación, 2004. (Technical Report 142-04) P. 125-134.

MANSETTO, M. Didática: a aula como centro. São Paulo: FTD, 1994.

MEdeiros, M.F.; MEdeiros, G.M.; COLLA, A.L.; HERRLEIN, M.B.P. A Produção de um Ambiente de Aprendizagem em Educação a Distância com o Uso de Mídias Integradas: a PUCRS virtual. 2001. Disponível em: <http://www.ead.pucrs.br/biblioteca/artigo/Abed_AmbientesAprendizagem.pdf>Acesso em: 12 maio 2011.

MORAN, J.M. A Educação que Desejamos: novos desafios e como chegar lá. Campinas, SP: Papirus, 2007.

MORAN, J.M.; MASETTO, M.; BEHRENS, M. Novas Tecnologias e Mediação Pedagógica. 16. ed. Campinas: Papirus, 2009.

NATALIE, H.N. Teaching Large Multilevel classes. Cambridge: Cambridge University Press, 2001.

NASS, C.; LEE, K.M. Does computer-generated speech manifest personality? an experimental test of similarity-attraction. In: SIGCHI CONFERENCE ON HUMAN FACTORS IN COMPUTING SYSTEMS - CHI '00, 2000, New York. Proceedings. [S.I.]: ACM, 2000. P. 329-336.

NUNES, Marcos Freitas ; BORGES, Thyago ; Alexander Vinson ; LOH, S. . Técnica para Analisar a Evolução do Perfil do Usuário com Base nas Suas Publicações. In: Escola Regional de Banco de Dados, 2007, Caxias do Sul. Anais da Escola Regional de Banco de Dados. Caxias do Sul : Universidade de Caxias do Sul, 2007. p. $132-140$. 
OLIVEIRA, M.K. Pensar a Educação: contribuições de Vygotsky. In: CASTORINA, J.A. et al. (Org.). Piaget, Vygotsky: novas contribuições para o debate. São Paulo: Ática, 1998. P. 51-83.

OLIVEIRA, M.K. Vygotsky: aprendizado e desenvolvimento: um processo sócio-histórico. São Paulo: Scipione, 1997.

PORFIRO, R.M. Uma Ferramenta Para Gestão de Grupos por Perfil de Alunos no Ambiente Moodle. 2008. Trabalho de Conclusão - Curso de Bacharelado em Sistemas de Informação, Faculdade de Informática, Pontifícia Universidade Católica do Rio Grande do Sul, 2008, Porto Alegre, BR-RS. Disponível em: <http://www. scribd.com/doc/4605923/Estudando-o-moodle> Acesso em: 30 maio 2011.

RESNICK, R. Recommender systems. Communications of the ACM, New York, v. 40, no. 3, p. 56-58, 1997.

SANTOS, O.C.; BOTICARIO, J.G. Building a knowledge-based recommender for inclusive eLearning scenarios. In: INTERNATIONAL CONFERENCE ON ARTIFICIAL INTELLIGENCE IN EDUCATION - AIED 2009, 14. , 2009, Brighton, UK. Proceedings. [S.I.]: IOS Press, 2009. P. 689-691.

SANTOS, O.C.; BOTICARIO, J.G. Users' experience with a recommender system in an open source standard-based learning management system. In: SYMPOSIUM OF THE WG HCI\&UE OF THE AUSTRIAN COMPUTER SOCIETY ON USABILITY \& HCI FOR EDUCATION AND WORK - USAB 2008, 4., 2008, Graz, Austria. Proceedings. [S.I.]: Springer, 2008. (Lecture Notes in Computer Science, 5298) P. 185-204.

SCHILLING, D.T. Teaching Classes of Mixed Ability Students. 2007. Disponível em: <http://www.tesolonline.com/articles/complete_articles.php?index=575\&category=103> Acesso em: 12 fevereiro 2011.

SHARDANAND, U.; MAES, P. Social information filtering: Algorithms for automating "word of mouth". In: HUMAN FACTORS IN COMPUTING SYSTEMS CONFERENCE - ACM CHI 95, 1995, Denver, Colorado. Proceedings. [S.I.]: ACM, 1995. P. 210-217. Disponível em: <http://www.csee.umbc.edu/ msmith27/readings/public/shardanand-1995a.pdf> Acesso em: 15 maio 2011.

TELEDUC: educação a distância. [Campinas]: UNICAMP/NIED, 2008. Disponível em: <http://www.teleduc. org.br/> Acesso em: 20 fev. 2011.

TERVEEN, L.; HILL, W. Beyond Recommender Systems: Helping People Help Each Other. In: CARROLL, J. (Ed.). Human-Computer Interaction in the Millennium. [S.I.]: Addison-Wesley, 2001. 21 pages.

THARP, Roland. G.; GALLIMORE, R. Rousing Minds to Life: Teaching, Learning, and Schooling in Social Context. Cambridge: Cambridge University Press (March 29, 1991). Disponível em: http://www.ncrel.org/sdrs/ areas/issues/students/learning/Ir1zpd.htm Acesso em: 20 março 2011.

TIFFANY, Y.T.; MCCALLA, G. Mining Implicit Ratings for Focused Collaborative Filtering for Paper Recommendations. In: WORKSHOP ON USER AND GROUP MODELS FOR WEB-BASED ADAPTIVE COLLABORATIVE ENVIRONMENTS - UM'03, 2003, Johnstown, USA. Proceedings. [S.I.: s.n.], 2003. Disponível em: http:// www.ia.uned.es/ elena/um03-ws/ Acesso em: 15 maio 2011. 
TIFFANY, Y.T.; MCCALLA, G. Smart Recommendation for an Evolving E-Learning System. International Journal on E-Learning, Norfolk, v. 4, no. 1, p. 105-129, 2005.

TRIVIÑOS, A.N. Introdução à Pesquisa em Ciências Sociais: a pesquisa qualitativa em educação. São Paulo: Atlas, 1987.

VIGOTSKY, L.S. A Formação Social da Mente: o desenvolvimento dos processos psicológicos superiores. São Paulo: Martins Fontes, 1998.

VIGOTSKY, L.S. Pensamento e Linguagem. São Paulo: Martins Fontes, 1989.

VIGOTSKY, L.S.; LURIA, A. R.; LEONTIEV, A.N. Linguagem, Desenvolvimento e Aprendizagem. São Paulo: Ícone, 1988.

WRIGHT, S.P. et al. Teacher and Classroom Context Effects on Student Achievement: Implications for Teacher Evaluation Journal of Personnel Evaluation Journal of Personnel Evaluation in Education, Dordrecht, no. 11 , p. 57-67, 1997.

YIN, R.K. Estudo de Caso: planejamento e métodos. Porto Alegre: Bookman, 2008.

ZAINA, L.A.M. Avaliação do Perfil do Aluno Baseado em Interações Contextualizadas Para Adaptação de Cenários de Aprendizagem. 2008. Tese (Doutorado) - Departamento de Engenharia de Computação e Sistemas Digitais, Escola Politécnica da Universidade de São Paulo, 2008, São Paulo, BR-SP.

Recebido em maio de 2011.

Aprovado para publicação em julho de 2011.

\section{Carlos Tadeu Queiroz de Morais}

Programa de Pós-Graduação em Informática da Educação e Programa de Pós-Graduação em Ciência da Computação, Universidade Federal do Rio Grande do Sul - UFRGS, Porto Alegre/RS, Brasil.

E-mail: cmorais@cinted.ufrgs.br

\section{Sérgio Roberto K. Franco}

Programa de Pós-Graduação em Informática da Educação e Programa de Pós-Graduação em Ciência da Computação, Universidade Federal do Rio Grande do Sul - UFRGS, Porto Alegre/RS, Brasil.

E-mail: sergio.franco@ufrgs.br 